\title{
Der Einfluss des Bildaufbaus in Narrativen auf die Erzählkomplexität von Vorschulkindern - eine empirische Vergleichsstudie
}

\author{
The Impact of Image Structure in Narratives on Story Complexity by \\ Pre-school Children - an Empirical Comparative Study
}

\section{Oliver Weber}

Humboldt-Universität zu Berlin, Deutschland

\begin{abstract}
The aim of the present study was to test the influence of picture composition on the narrative complexity of preschool children, and to compare the different procedures of the Cat Story of Hickmann (2002) and the Fox Story of Gülzow \& Gagarina (2007) with the Baby Birds and Baby Goats Story of MAIN, by Gagarina et al. (2012). For this purpose, 27 children between the ages of 5;01 and 6;09 were tested with both variants to check whether a macro-structurally controlled picture structure would lead to more complex stories. The results show that narratives with a Goal-Attempt-Outcome structure, i.e. the Baby Birds and Baby Goats Stories, make children with increasing age tell more complex stories by means of a rise in story complexity than the narratives of Hickmann and Gülzow \& Gagarina without that structure.
\end{abstract}

\section{$1 \quad$ Einführung}

Geschichten, die Kinder erzählen, geben Aufschluss über ihre kognitiven, semantischen und sozialen Fähigkeiten. Gerade für den späteren Erfolg in der Schule spielt die Herausbildung narrativer Fähigkeiten bei Vorschulkindern eine wichtige Rolle. Bereits erworbene Kompetenzen zu Handlungszusammenhängen und Gesprächsverläufen können Kinder sowohl beim Erwerb ihrer Schriftlichkeit als auch ihrer Gesprächskompetenz unterstützen. Diese wiederum hat wesentlichen Einfluss auf die Sprachentwicklung und die Lesekompetenz. Um Kinder auf ihre narrativen Fähigkeiten hin zu untersuchen, gibt es neben einer Vielzahl von Testvarianten das Verfahren, semi-spontane Sprachdaten des Kindes zu erhalten, indem man ihm Bildergeschichten zeigt und es diese nacherzählen lässt. Von diesen Bildergeschichten existieren unterschiedliche Verfahren. 
Die Cat Story (CAT; Hickmann, 2002) entstand im Rahmen von Hickmanns Studie zu kindlichem Diskurs und deren Organisation von Handlungssequenzen in Bezug auf Kohärenz und Kohäsion. Die Fox Story (FOX; Gülzow \& Gagarina, 2007) wurde von Insa Gülzow und Natalia Gagarina konzipiert, um anaphorische Referenzen zu untersuchen, die bilinguale Kinder in den Sprachen Deutsch, Russisch und Bulgarisch verwenden. Die Baby Birds (BB) und Baby Goats $(B G)$ Geschichten (Gagarina et al. 2012) haben das Ziel, die Erzählfähigkeiten von Kindern zu erforschen. Alle diese Bildergeschichten bestehen aus jeweils sechs Bildern, die einen Handlungsverlauf zeigen. Sie unterscheiden sich aber hinsichtlich des Hintergrunds ihrer Entstehung und in Bezug auf ihre Makrostruktur, ihr Format und die Testdurchführung voneinander.

Auf Ebene der Makrostruktur, welche im Fokus der Untersuchung steht, sind $B B$ und $B G$ mit einem kontrollierten Bildkonzept ausgestattet, der GoalAttempt-Outcome-Struktur (GAO). Die Protagonisten der Geschichte haben jeweils ein Ziel, das sie erreichen wollen und sie unternehmen Versuche, dieses Ziel zu erlangen, welche zu messbaren Ergebnissen führen. Die Geschichten werden als bunte Bilder in Form eines zusammenhängenden Handlungsstrahls präsentiert. CAT und FOX haben keine kontrollierte Makrostruktur und erscheinen als schwarz-weiße Zeichnungen auf einzelnen Kartonkarten, die dem Kind vorgelegt werden.

Es existieren folglich Sprachdaten von Kindern, welche sowohl $C A T$ und $F O X$ erzählt haben, als auch Aufzeichnungen zu $B B$ und $B G$. Es gibt aber keine Vergleichsdaten darüber, dass Kinder mit beiden Narrativen getestet wurden. Demnach können die Ergebnisse der einen Studie nicht unbedingt mit denen der anderen verglichen werden. Das Anliegen dieser empirischen Studie ist es daher, in einer Testreihe Vorschulkinder beide Varianten der Bildergeschichten erzählen zu lassen und die Ergebnisse miteinander zu vergleichen. Die Untersuchungen sollen den Einfluss eines makrostrukturell kontrollierten Bildes auf die Produktion und Perzeption von Narrativen in einer Vergleichsstudie prüfen.

Alle makrostrukturellen Parameter der in dieser Studie untersuchten Narrative können Einfluss auf die von den Kindern produzierten Geschichten nehmen. Die Konzeption einer GAO-Struktur impliziert eine messbare Veränderung in der Produktion und Perzeption von Narrativen. Wie reagieren die getesteten Kinder auf die unterschiedlichen bildlichen Stimuli? Die Daten werden hinsichtlich unterschiedlicher Parameter untersucht: Neben einer Gegenüberstellung der beiden Geschichten mit $G A O$-Struktur und den beiden Geschichten ohne diese Struktur werden Testtermin, das Alter der Probanden und das Geschlecht berücksichtigt.

Als erstes werden im folgenden Kapitel die besagten Bildergeschichten vorgestellt. Daran anschließend werden die vier Testkategorien des Multilingual Assessment Instrument for Narratives (MAIN) betrachtet, da sie für diese Studie 
und die Analyse der Daten entscheidend sein werden. Bevor die Testergebnisse vorgestellt und analysiert werden, stehen die theoretischen und praktischen Vorbereitungen dieser Studie zur Diskussion.

\section{Der Gebrauch von Narrativen und ihre Varianten}

Zunächst werden die genannten vier Bildergeschichten vorgestellt, welche dazu herangezogen werden, kindliche Sprache hinsichtlich unterschiedlichster Fragestellungen zu untersuchen. Dies ist Hickmanns (2002) Cat Story (CAT), die Fox Story (FOX) von Gülzow \& Gagarina (2007) und die Baby Birds (BB) und Baby Goats (BG) Geschichten des MAIN von Gagarina et al. (2012). Die unterschiedlichen Termini „Story“ und „Geschichte“ deuten bereits eine Dichotomie an, die im Laufe des Kapitels näher erläutert wird. Im Zusammenhang mit der Eingangsfrage nach dem Einfluss eines makrostrukturell kontrollierten Bildaufbaus auf die Erzählfähigkeiten von Kindern stehen $C A T$ und $F O X$ den $B B$ und $B G$ Geschichten gegenüber. Letztere weisen durch ihre Bildkonstruktion eine Goal-Attempt-Outcome-Struktur auf, die nach den Prinzipen der Story Grammar und Story Structure, sowie Westby's (2005) Binary Decision Tree erstellt ist.

\subsection{Cat Story}

In ihrer Studie zu kindlichem Sprachgebrauch stellt Hickmann (2002) zwei Bildergeschichten vor, die jeweils aus schwarz-weißen Zeichnungen ohne Text bestehen. Eine davon ist CAT (siehe Abbildung 1), die eine Vogelfamilie zeigt. Die Vogelmutter verlässt das Nest und kommt erst am Ende der Geschichte zurück. Die Küken bleiben alleine zurück. Die Katze erscheint bereits im zweiten Bild und bleibt bis zum Ende der Geschichte die Hauptfigur. Mit dem Erscheinen des Hundes ändert sich allerdings ihr Status vom Agens zum Patiens der Handlung. Die Geschichte weist zudem eine komplexe zeitliche Organisation auf. Handlungen folgen in direktem zeitlichen Anschluss oder überlappen, wie bereits auf Bild 2. Die Ankunft der Katze geschieht zum selben Zeitpunkt, wie das Davonfliegen der Vogelmutter. Eine weitere zeitliche Parallelität ereignet sich auf Bild 4, das sowohl das Erscheinen des Hundes, als auch den Versuch der Katze, auf den Baum zu klettern, zeigt. Bild 5 zeigt die zurückkommende Vogelmutter und die Situation, dass der Hund die Katze vom Baum zieht (Vgl. Hickmann, 2002:184). 

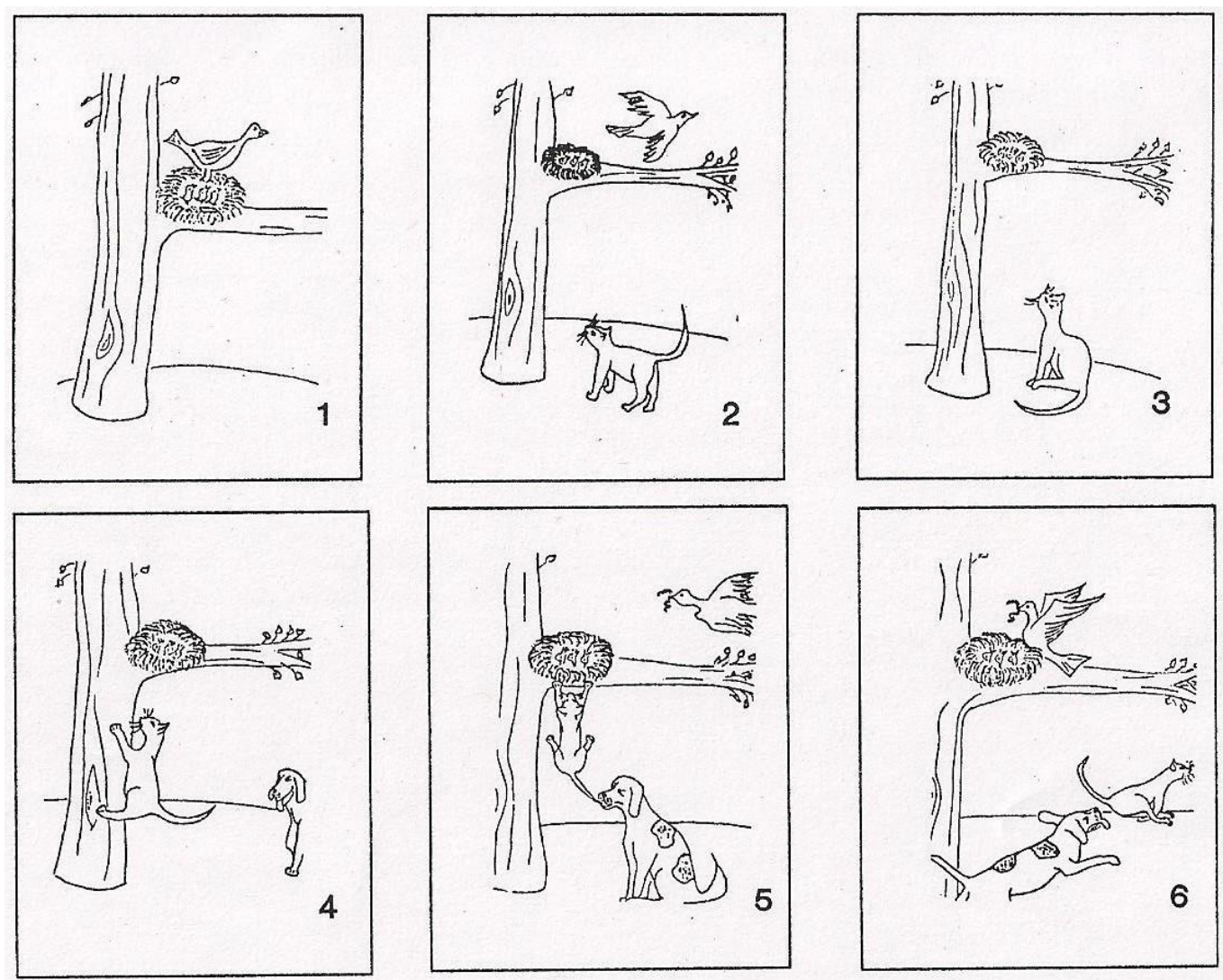

Abbildung 1: Cat Story (Hickmann. 2002:344)

Das Testverfahren sieht vor, die Kinder einzeln zu testen. Hickmann (2002:185) nennt es ein ,story-telling game“, bei dem Kinder die Geschichte einer Person erzählen, welche die Geschichte nicht kennt und auch die Bilder nicht sieht. Dazu schaut sich das Kind die ganze Geschichte an, um sie anschließend so genau wie möglich der Kontrollperson zu erzählen. Hierbei darf die Kontrollperson keine suggestiven, sondern nur generelle Fragen stellen, wie z.B. „Was geschieht?“ (Vgl. Hickmann, 2002:185).

Die Tests finden in Erziehungseinrichtungen statt. Je nach Alter der Kinder ist dies der Kindergarten, die Vor- oder Grundschule des Kindes (Hickmann, 2002:185).

\subsection{Fox Story}

FOX entstand im Rahmen der Studie "Noun Phrases, Pronouns and Anaphoric Reference in Young Children Narratives" (Gülzow \& Gagarina, 2007). Das Ziel war die Untersuchung anaphorischer Referenzen, die bilinguale Kinder in den Sprachen Deutsch, Russisch und Bulgarisch verwenden.

Die Geschichte (siehe Abbildung 2) besteht aus sechs einzelnen Bildern in schwarz-weißer Strichzeichnung (Vgl. Kuehnast, 2006). Die Protagonisten von FOX sind nach dem Kriterium ihres grammatikalischen Genus ausgewählt. Diese 
sind ein Vogel, ein Fisch und ein Fuchs, die im Deutschen jeweils maskulines Genus aufweisen, im Russischen und Bulgarischen hingegen feminin sind. Dadurch kann der Gebrauch von Demonstrativ- und Personalpronomina, sowie unterschiedliche Möglichkeiten von Nominalphrasen getestet werden (Vgl. Gülzow \& Gagarina, 2007:209).

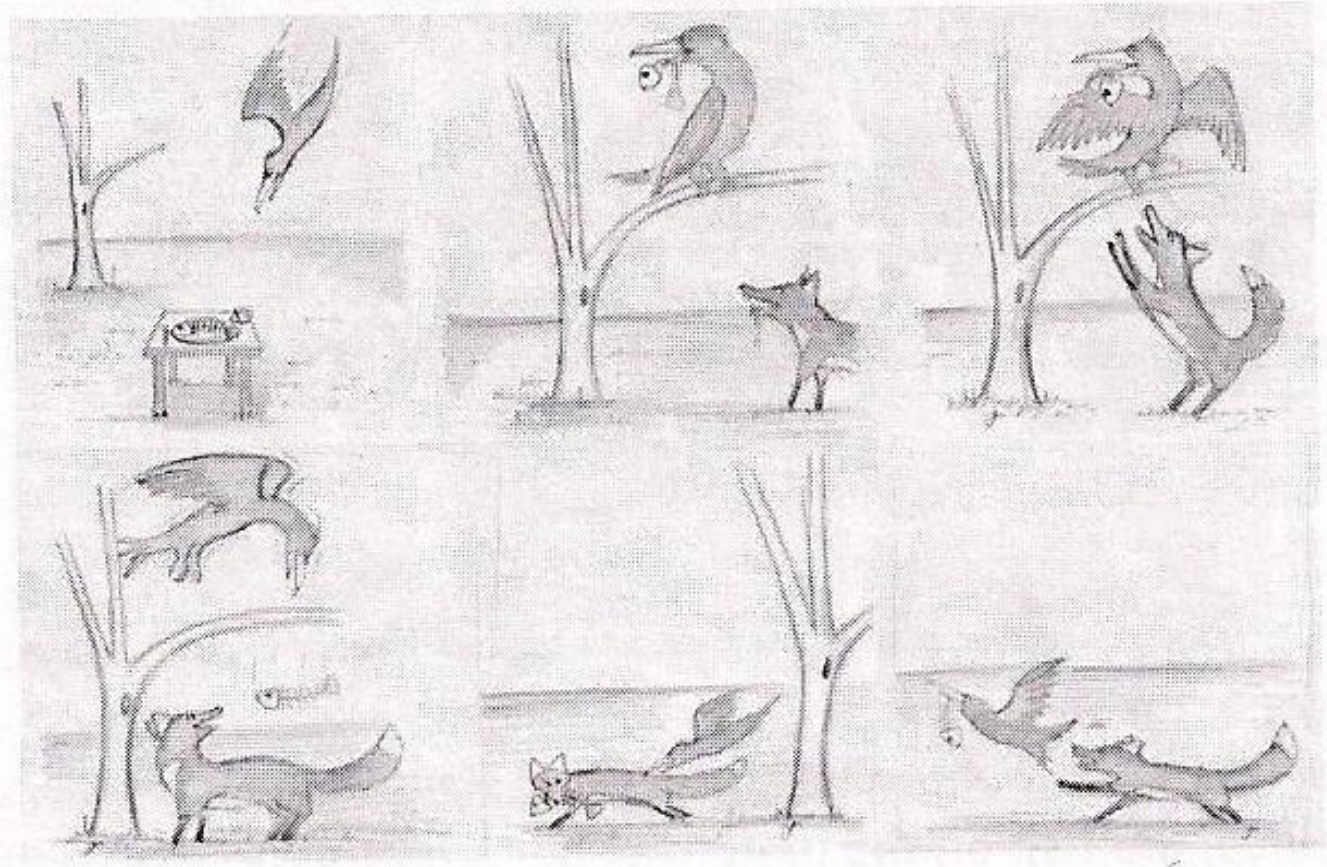

Abbildung 2: Fox Story (Gülzow \& Gagarina. 2007. 209)

Die Geschichte berichtet von einem Vogel, der einen Fisch (bzw. eine Fischgräte) gefunden hat und einem Fuchs, der diesen Fisch ebenfalls haben möchte. Im Verlauf der Geschichte wechselt der Fisch drei Mal den Besitzer. Es gibt also drei Protagonisten, wobei der Fisch ausschließlich Patiens des Geschehens bleibt.

Der Testverlauf schreibt vor, den Kindern nicht alle Bilder auf einmal zu zeigen, sondern immer nur zwei zur gleichen Zeit. Dieses Verfahren ermöglicht die Untersuchung anaphorischer Relationen (Vgl. Kuehnast, 2006:127). Um das Kind zu ermutigen, die Geschichte zu erzählen, ist die Kontrollperson angehalten, keine gezielten Fragen nach den Protagonisten zu stellen, sondern nach dem Geschehen und dem Fortgang der Geschichte zu fragen (Vgl. Gülzow \& Gagarina, 2007:209).

\subsection{Baby Birds Geschichte}

$B B$ ist das Ergebnis einer Bearbeitung von $C A T$. Bei der Erstellung der Makrostruktur haben sich die Autoren (Gagarina et al., 2012) an dem Prinzip der Story Grammar (Stein \& Glenn, 1979) orientiert. Die Geschichten sind so 
konzipiert, dass folgende Komponenten der Makrostruktur (außer dem Setting) jeweils dreimal in einer Geschichte vorkommen:

- Eine Situation (Setting), die auf Zeit und Ort der Handlung verweist und nicht innerhalb einer Episode vorkommt.

- Ein innerer Gemütszustand (Internal State Term = IST), der zum Auslöser der folgenden Geschichte wird.

- Das Ziel (Goal) ist die Planung des Protagonisten, mit einer vorhandenen Situation umzugehen.

- Der Versuch (Attempt) zeigt eine Aktion, die das Ziel zu erreichen verspricht.

- Das Resultat (Outcome) ist das erfolgreiche oder auch misslungene Ergebnis des vorangegangenen Versuchs.

- Der IST (Internal State Term) als Reaktion verdeutlicht die Gefühle oder Gedanken des Protagonisten, die das Resultat mit sich bringt (Gagarina et al., 2012:19).

Konkret bedeutet das für $B B$, dass im Setting Angaben zu Zeit und Ort gemacht werden, wie beispielsweise: „Es war einmal in einem Wald eine Vogelmutter mit ihren Küken." Der IST als einleitendes Ereignis könnte für die erste Episode lauten: „Die Vogelmutter sieht, dass ihre Kleinen hungrig sind.“ In der zweiten Episode sieht die Katze, wie die Vogelmutter davonfliegt. Die Katze könnte ebenso mit dem Attribut ,hungrig' eingeführt werden. Daraus resultiert das folgende Verhalten der Katze. In der dritten Episode beobachtet der Hund, dass die Küken in Gefahr sind, weil die Katze nach ihnen greift. Diese Einschätzung ist ebenfalls Ausgangspunkt für die Reaktion des Hundes. $\mathrm{Zu}$ allen drei Protagonisten von $B B$ kann also ein einleitender Gemütszustand beschrieben werden, der als Auslöser für das weitere Vorgehen dient.

Goal: Das Ziel des Vogels ist es, Futter für die Küken zu besorgen. Die Katze möchte die kleinen Vögel fressen, der Hund will sie davon abhalten und die Küken retten.

Attempt: Als Versuch des Vogels kann gewertet werden, dass dieser davonfliegt, um Futter zu suchen. Die Katze klettert auf den Baum, um an die Küken zu gelangen und der Hund beißt der Katze in den Schwanz, um dies zu verhindern.

Outcome: Das Resultat der Futtersuche des Vogels ist der mitgebrachte Wurm. Die Katze hat (für kurze Zeit) einen der kleinen Vögel in ihren Krallen. Der Hund verjagt die Katze.

IST: Eine mögliche IST als Reaktion der ersten Episode ist eine zufriedene Vogelfamilie. Für einen kurzen Moment ist die Katze glücklich und der Hund in 
der dritten Episode stolz, dass er die Katze verjagt hat und dadurch die Vögel gerettet hat (Gagarina et al., 2012:119-120).

\subsection{Baby Goats Geschichte}

Die makrostrukturellen Komponenten von $B G$ beginnen ebenfalls mit einem Setting. Das Testprotokoll schlägt dafür wieder die Einleitung „Es war einmal“ oder auch „Eines Tages“, sowie „Vor langer Zeit“" vor. Ort der Handlung ist eine Wiese, ein Wald, an einem See oder ähnliches. Der einleitende Gemütszustand der Ziegenmutter und ihres Zickleins kann mit ängstlich oder auch sorgenvoll benannt werden. Die Ziegenmutter sieht, dass eines ihrer Zicklein im Wasser zu ertrinken droht. Der Fuchs in Episode zwei ist hungrig und bemerkt, dass eines der beiden anderen Zicklein unbeaufsichtigt ist. Der Vogel sieht, dass diese kleine Ziege in Gefahr ist.

Goal: Das Ziel der Ziegenmutter ist es, ihrem kleinen Zicklein aus dem Wasser zu helfen. Der Fuchs will in Episode zwei das Zicklein fangen, doch der Vogel beschließt in Episode drei das Zicklein zu retten.

Attempt: Die Ziegenmutter versucht, das Zicklein aus dem Wasser zu schieben. Der Fuchs springt nach vorne und will das Zicklein fangen. Der Vogel attackiert den Fuchs und beißt ihm in den Schwanz.

Outcome: Das Resultat ihres Versuchs ist, dass die Ziegenmutter ihr Kleines aus dem Wasser gerettet hat. Der Fuchs hat in Episode zwei das Zicklein gefasst und der Vogel hat den Fuchs schließlich verjagt.

IST: Die emotionale Reaktion der Ziegenmutter in der ersten Episode ist, dass sie glücklich darüber ist, ihr Junges gerettet zu haben. Der Fuchs freut sich, dass er das Zicklein gefangen hat und der Vogel ist erleichtert oder stolz, dass er das Ziegenbaby gerettet hat (Gagarina et al., 2012:125-126).

Die Bildergeschichten zeigen jeweils klar dargestellte Handlungen. Die Intentionen der Protagonisten sind sorgfältig herausgearbeitet, sowie ihre emotionale Haltung durch Gestik und Mimik erkennbar. Jede Geschichte ist mit drei Episoden ausgestattet, um dem Kind mehrere Möglichkeiten zu geben, eine Geschichte in Form von einleitenden Ereignissen, Goals, Attempts und Outcomes sowie emotionale Reaktionen zu erzählen. Diese Gestaltung ermöglicht es, die strukturelle Komplexität, der vom Kind erzählten Geschichte genau identifizieren zu können (Gagarina et al., 2012:125-126).

\section{Das Multilingual Assessment Instrument for Narratives}

Vor dem Hintergrund der Analysen über metakognitive Kompetenzen in Bezug auf die Sprachentwicklung von Kindern entstand am Zentrum für Allgemeine 
Sprachwissenschaft in Berlin 2012 das Multilingual Assessment Instrument for Narratives, kurz MAIN (Gagarina et al. 2012). Dieses Testverfahren ist geeignet, um sowohl die Produktion als auch die Perzeption von Narrativen zu erfassen. Die Bilder der Geschichten sind hinsichtlich ihrer kognitiven und linguistischen Komplexität, sowie der Parallelität von Mikro- und Makrostruktur kontrolliert (Vgl. Gagarina et al., 2012:1).

Der Test ist in vier Teilbereiche untergliedert, wobei jeder dieser Bereiche separate Kompetenzen der Kinder fokussiert. Dadurch kann gezielt der Erzählungsaufbau analysiert oder das Verständnis getestet werden.

\subsection{Story Structure}

Alle Geschichten verfügen über ein Setting und eine Episodenstruktur, wie sie z.B. von Thorndyke (1977), Stein \& Glenn (1979) und Westby (2005) vorgestellt wurden. Diese Arbeiten bilden die theoretischen Rahmenbedingungen für die Konstruktion der Bildergeschichten, die Gagarina et al. im Zusammenhang der MAIN-Studie entwickelt haben. Außerdem hat man sich am Diskurs-AnalyseModell von Trabasso und Nickels (1992) orientiert. Dieses klassifiziert den Inhalt der Geschichten in sechs Kategorien, mit dem ein Hierarchieplan erstellt wird (Vgl. Trabasso \& Nickels, 1992:252).

In Bezug auf $B B$ und $B G$ der $M A I N$-Studie wurden diese Kategorien übernommen. Die makrostrukturelle Analyse beginnt bei der Episode. Jede Bildsequenz zeigt drei kurze Episoden, welche dem Kind drei Möglichkeiten eröffnen, über einleitende Ereignisse, Ziele (Goals), Lösungsversuche (Attempts) und Ergebnisse (Outcome) zu berichten (Vgl. Gagarina et al., 2012:11). Jede Episode wiederum besteht aus einem Ziel des Protagonisten, einem Versuch, dieses Ziel zu erreichen, einem Ergebnis und mentalen Zuständen, die mit dem Ziel in Verbindung stehen und die Reaktionen ausdrücken (Gagarina et al., 2012:11).

\subsection{Structural Complexity}

Eine Analyse der strukturellen Komplexität von erzählten Bildergeschichten liefert Informationen über die Erzählkompetenz des Kindes, auf welcher der Fokus dieser Studie liegt. Bei der Auswertung der Ergebnisse wird besondere Aufmerksamkeit auf die Anzahl der produzierten GAO-Strukturen gelegt. Die Fähigkeit, sorgfältig ausgearbeitete Episoden zu produzieren, erfordert ein Verständnis narrativer Schemata, von Kausalzusammenhängen, der Fähigkeit, des Anderen Perspektive einzunehmen, das metakognitive Bewusstsein der Planung und das Bedürfnis, Handlungen zu vollenden. Für $B B$ und $B G$ sind, in 
Anlehnung an Westby's Binary Decision Tree (Westby, 2005:181), drei Stufen der Komplexität erstellt worden:

1) Sequenzen, die kein Ziel (Goal) beinhalten;

2) Unvollständige Episoden, die zwar ein Ziel, aber weder Lösungsversuch (Attempt) noch Ergebnis (Outcome) enthalten und somit keine komplette $G A O$-Struktur aufweisen;

3) Vollständige Episoden, die alle drei Komponenten der $G A O$-Struktur beinhalten (Vgl. Gagarina et al., 2012:11).

Um diese Goal-Attempt-Outcome-Struktur haben Gagarina et al. (2012) in ihrem Decision Tree das Geschichtsschema von Westby erweitert:

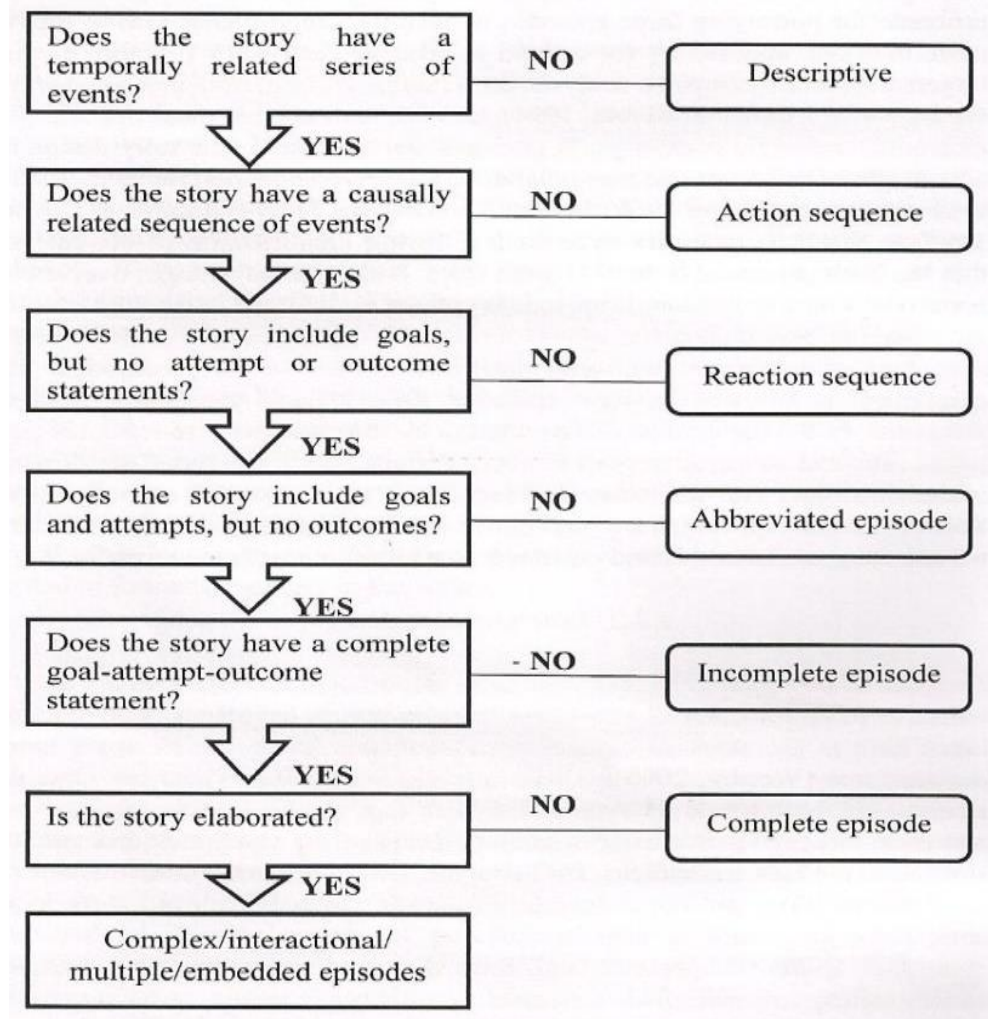

Abbildung 3: Decision Tree (Gagarina et al., 2012:12)

Wenn die Bildsequenz keine ausreichenden Informationen bietet, um die Ziele des Protagonisten zu erkennen und darüber hinaus den kausalen und zeitlichen Zusammenhang zwischen Goals, Attempts und Outcomes herzustellen, wird keine kohärente Geschichte daraus resultieren - unabhängig von den metakognitiven Fähigkeiten des Kindes (Vgl. Trabasso \& Nickels, 1992:252). 


\subsection{Internal State Terms}

Um eine Geschichte zu verstehen, müssen die Emotionen, Ziele und Absichten der Protagonisten interpretiert werden. Diese internen und mentalen Zustände sind im Zusammenhang der Theory of Mind (ToM) Fähigkeiten, wie IntentionReading und Perspective-Taking, erforscht worden (Vgl. Tomasello, 2003). Eine Analyse der produzierten IST bei Bildergeschichten kann Hinweise auf die ToMFähigkeiten von Kindern liefern. Die Benutzung von metalinguistischen und metakognitiven Verben, sowie der Gebrauch von Emotionsausdrücken zeigt, dass Kinder sich in andere hineinversetzen können. Dadurch sind sie in der Lage, Zusammenhänge herzustellen und Absichten zu interpretieren (Vgl. Westby, 2005:177-181). In Bezug auf die MAIN-Studie wird der Gebrauch von IST dahingehend untersucht, ob Kinder das zielgerichtete Verhalten des Protagonisten verstehen. Außerdem werden sie herangezogen, um das Verständnis der Geschichte und die Schlussfolgerungen der Kinder auf emotionale Fragen zu testen (Vgl. Gagarina et al., 2012:14).

\subsection{Comprehension}

Um zu untersuchen, inwieweit Kinder die wichtigsten Komponenten der Geschichtsstruktur verstehen, schließt der Test mit zehn Fragen ab. Drei dieser Fragen richten sich an die drei Hauptziele der Geschichte, sechs weitere Fragen behandeln IST, die entweder im Zusammenhang mit einleitenden Ereignissen oder Reaktionen stehen. Sie erforschen das Urteilsvermögen der Kinder. Die letzte Frage analysiert eine ToM-Schlussfolgerung und zeigt, ob das Kind die Geschichte als Ganzes verstanden hat (Gagarina et al., 2012:14-15).

Das MAIN bietet die Möglichkeit, die Erzählkompetenzen von mono- und bilingualen Kindern gezielt zu evaluieren. Das Ergebnis der makrostrukturellen Bearbeitung der Bilder soll alle Informationen liefern, um die Goals, Attempts und Outcomes der einzelnen Episoden zu verstehen und diese wiedergeben zu können (Gagarina et al., 2012:20).

Die Gestaltung der vorgestellten vier Bildergeschichten ist das Resultat divergierender Forschungsschwerpunkte. Aufgrund der Frage, ob Kinder abweichende Geschichten hinsichtlich ihrer strukturellen Komplexität produzieren, je nachdem, welche der vorgestellten Geschichten sie nacherzählen, müssen Testverfahren und Bildformat einander angeglichen werden. Dies wird in den folgenden Kapiteln detailliert beschrieben. 


\section{Die theoretischen und praktischen Vorbereitungen der Studie}

Da es, wie zuvor beschrieben, unterschiedliche Varianten von Narrativen gibt, die dazu herangezogen werden, die Erzählfähigkeiten von Kindern zu untersuchen, muss zunächst gewährleistet werden, dass die Unterschiede, welche die Ergebnisse hervorbringen, auf die Makrostruktur der Bilder zurückzuführen sind. Daher soll zunächst der Frage nachgegangen werden, welchen Einfluss die Farbgestaltung der Bilder auf die Erzählkomplexität der von den Kindern erzählten Geschichten hat. Im Anschluss daran wird die Modifizierung der Testdurchführung und der neu erstellte Bewertungsbogen für FOX erläutert, bevor die Testpersonen vorgestellt werden.

\subsection{Die Auswirkungen der Farbgestaltung von Narrativen}

Abgesehen vom divergierenden Testverfahren von $C A T$ und $F O X$ gegenüber $B B$ und $B G$ unterscheiden sich die Bildergeschichten bereits durch die Tatsache, dass erstere $(C A T \& F O X)$ schwarz-weiß und letztere $(B B \& B G)$ in Farbe vorliegen. Dadurch stellt sich zunächst die Frage, ob die Farbgestaltung von Narrativen einen Einfluss auf die Produktion und Perzeption bei Vorschulkindern ausübt. Schneider, Rivard und Debreuil (2011) haben herausgefunden, dass es keinen Unterschied zu verursachen scheint, wie die Farbgestaltung der Bildergeschichten konzipiert ist. Sie sind davon ausgegangen, dass farbige Bilder die Aufmerksamkeit von Kindern in höherem Maße erlangen, als die gleichen Bilder in schwarz-weiß. Dies hätte, so die Vermutung, Auswirkungen auf die Länge der von den Kindern erzählten Geschichte und würde sich in deren Komplexität (Story Complexity) niederschlagen (Schneider et al., 2011:372-373). Dazu wurden 22 Kinder im Alter zwischen 48,3 und 77,8 Monaten mit dem Edmonton Narrative Norms Instrument (ENNI; Schneider, Dubé, \& Hayward, 2005) getestet. Den Kindern wurde je eine farbige und eine schwarz-weiße Variante derselben Bildergeschichte vorgelegt. Weder im Bereich der Story Grammar, noch in der Anzahl der Worte kam die Studie zu einem signifikant unterschiedlichen Ergebnis zwischen den beiden Geschichtsvarianten (Schneider et al., 2011). Dem zufolge haben farbige Bilder keinen Einfluss auf die Produktion und Perzeption von Narrativen. Für die erhobenen Daten wurden Kinder im Alter zwischen 61 und 81 Monaten getestet. Damit überschneiden sich zu einem Großteil die Altersspannen der Probanden dieser Studie mit denen der oben genannten. Das Ergebnis der Farbstudie von Schneider, Rivard und Debreuil (2011) bedeutet somit, dass in der hier vorgenommenen Gegenüberstellung von $C A T$ und $F O X$ mit $B B$ und $B G$ der Unterschied von schwarz-weißen und farbigen Bildern vernachlässigt werden kann. Der Einfluss von Farbe bei 
Bildergeschichten auf die Erzählkompetenz von Vorschulkindern kann für diese Studie folglich ausgeschlossen werden.

\subsection{Testmodifizierung}

Um die Bildergeschichten miteinander vergleichen zu können, müssen zunächst das Format und der Testablauf modifiziert werden. Für dieses Experiment wird die Verfahrensweise der MAIN-Studie auf CAT und FOX übertragen. Dies geschieht, da das MAIN für $B B$ und $B G$ eine dezidiertere Beschreibung der Testvorbereitungen und des Verlaufs, sowie ein ausgearbeitetes Testprotokoll liefert. Dazu werden zunächst die einzelnen Bildkarten von $C A T$ und $F O X$ auf die gleichen Maße der Bilder von $B B$ und $B G$ verkleinert und anschließend auf einen gefalteten Kartonstreifen geklebt. Dies geschieht vor dem Hintergrund, dass $C A T$ und FOX dadurch kein Shared-Attention Procedure mehr ist und wie $B B$ und $B G$ nur vom Kind betrachtet werden kann. Ist dies der Fall, kann das Kind nicht auf die Verwendung von Deiktika zurückgreifen. Dadurch wird bereits der Unterschied zwischen Shared-Attention und Non-Shared-Attention aufgehoben.

Bevor das Kind seine Bildergeschichte erzählt, bekommt es eine Modellgeschichte erzählt. Währenddessen schaut es sich die Geschichte an. Dieses vorherige Erzählen einer Modellgeschichte ist weder bei CAT noch FOX vorgesehen. Um die Rahmenbedingungen jedoch so ähnlich wie möglich zu gestalten, wird beim ersten Testtermin allen Kindern die Modellgeschichte „Cat" vorgelesen und beim zweiten Termin die Modellgeschichte „Dog“.

Wie in den Anleitungen zur MAIN-Studie vorgeschrieben darf sich das Kind einen von den drei Umschlägen aussuchen, die sich auf dem Tisch befinden. Dieses Vorgehen kontrolliert den Shared-Knowledge-Effekt (Gagarina et al., 2012:6) während der Präsentation der Bildergeschichten und suggeriert dem Kind zum einen, dass auch die Kontrollperson nicht weiß, welche Geschichte es nun erzählen wird. Zum anderen sind Kinder motivierter, eine Geschichte zu erzählen, die sie sich selbst ausgesucht haben (Westby, 2005:198).

Der Protokollbogen für $B B$ kann ohne Einschränkung auf $C A T$ angewendet werden, da erstere aus letzterer hervorgegangen ist. Die Änderungen, welche für $B B$ vorgenommen wurden, haben keinen Einfluss auf die Evaluation. Die Bereiche Produktion (Story Structure, Story Complexity und IST) und Perzeption (Comprehension) können einschränkungsfrei angewendet werden.

\subsection{Bewertungsbogen Fox Story}

Im Gegensatz zu den Protokollen für $B B$ und $B G$ existiert keine Vorlage zu $F O X$. Daher wurde diese vom Autor für diese Studie erstellt. 
Im Abschnitt I Produktion wird die Story Structure (SS) bewertet. In Anlehnung an den Bewertungsbogen der MAIN-Studie kann auch bei FOX unter A 1. das Setting über Zeit und/oder Ort des Geschehens erfasst werden. Problematisch hingegen wird es bereits bei A 2., dem einleitenden Ereignis der ersten Episode „Vogel“. Im Gegensatz zu $B B$ und $B G$ enthält $F O X$ keine Ausgangssituation, die unabhängig von einer Aktion der Agentia und/oder Patientia konstatiert wird. Der Vogel (Agens) fliegt direkt auf die Fischgräte (Patiens) zu, ohne dass eine vorangegangene interne Motivation erkennbar ist. Im Flug zum Fisch auf Bild 1 vereinen sich bereits Goal (A 3.) und Attempt (A 4.) der ersten Episode. Diese können aber ggf. gesondert voneinander durch das Kind erzählt werden, beispielsweise wenn es berichtet, dass der Vogel den Fisch fressen wollte und deshalb zu ihm hinfliegt.

Auf dem zweiten Bild sieht man den Vogel auf einem Baum sitzend, mit der Fischgräte im Schnabel. Der Outcome (A 5.) der ersten Episode ist demnach, dass der Vogel den Fisch erfolgreich aufgegriffen hat. Als IST-Reaktion (A 6.) besteht die Möglichkeit, den Vogel als zufrieden, glücklich oder stolz zu bezeichnen. In Episode 1 kann das Kind bereits nur sechs Punkte erzielen und nicht, wie bei $C A T$ oder der $B B$ und $B G$, sieben.

Episode zwei „Fuchs“ kann identisch zu den Bewertungsvorgaben von $B B$ und $B G$ erstellt werden. Es gibt auf dem zweiten Bild ein einleitendes Ereignis, dem man den Gemütszustand des Fuchses entnehmen kann (Fuchs sieht Vogel/Fisch; Fuchs ist hungrig). Das Ziel des Fuchses ist es, die Fischgräte zu bekommen (Goal A 8.). Der Fuchs springt nach oben (Attempt A 9.), der Vogel lässt die Fischgräte fallen, bzw. der Fuchs besitzt nun die Fischgräte (Outcome A 10.) und ist zufrieden (IST A 11.). Episode zwei unterscheidet sich nicht von $B B$ oder $B G$ im Hinblick auf die zu erreichenden Punkte.

In der dritten Episode wird bei $F O X$ hingegen keine weitere Entität eingeführt, wie es der Fall des Hundes in $B B$, oder des Vogels in $B G$ ist. In $F O X$ wechselt die Fischgräte jedoch erneut ihren Besitzer. Dem geht allerdings kein einleitendes Ereignis voraus. Im direkten Anschluss auf Bild 4, das zeigt, wie die Fischgräte aus dem Schnabel des Vogels fällt, sieht man auf Bild 5, dass der Fuchs diese im Maul hat und davonläuft. Der Vogel hat bereits die Verfolgung aufgenommen. In Bild 5 vereinen sich demnach Outcome (A 10.) der zweiten Episode, sowie Goal (A 13. Vogel wollte Fisch zurückhaben) und Attempt (A 14. Vogel beißt Fuchs; Vogel fliegt hinter Fuchs her; o.ä.) der dritten Episode. Das letzte Bild (Bild 6) zeigt den Vogel, wie er mit der Fischgräte im Schnabel vor dem Fuchs davonfliegt und der diesem hinterherläuft. Als Outcome (A 15.) der dritten Episode kann gewertet werden, dass der Vogel den Fisch wieder/zurückhat und die $I S T$ als Reaktion ist, dass der Vogel zufrieden und der Fuchs immer noch hungrig ist. Dennoch kann das Kind im ersten Abschnitt unter $S S$ nur fünfzehn, 
und nicht wie bei $B B$ und $B G$ siebzehn Punkte erreichen. Die Möglichkeit, eine komplette $G A O$-Sequenz zu produzieren, hat es dennoch in allen Geschichten.

Somit kann Abschnitt I, B. Structural Complexity (SC) ohne Veränderungen oder Einschränkungen übernommen werden. Ebenso die Erfassung der unter Abschnitt I, C. IST aufgelisteten physiologischen und emotionalen Adjektive, sowie der perzeptuellen, mentalen und linguistischen Verben. Im Abschnitt II: Verständnis können bei $F O X$, genau wie bei $B B$ und $B G$, zehn Fragen an das Verständnis des Kindes gestellt werden.

D 1. „Warum fliegt der Vogel zu dem Fisch?“ ist direkt abgeleitet von der Frage „Warum fliegt die Vogelmama weg?“ in $B B$ oder der Frage „Warum war die Ziegenmutter im Wasser?" in $B G$. Da der Vogel in $F O X$ alleine jagt, kann nicht nach dem Befinden seiner Jungen gefragt werden, wie es unter D 2. „Wie fühlen sich die Vogelbabys?" bzw. „Wie fühlt sich das Ziegenkind?“" geschieht. Als Modifikation wird bei FOX nach dem Befinden des Vogels selbst gefragt. Als richtige Antwort gelten hier die Varianten „hungrig“ o.ä., wohin gegen die Aussage „gut“, „glücklich“ oder „will spielen“ als falsch bewertet wird. Dementsprechend kann unter D 3. nach dem „Warum denkst du...“ gefragt werden. Die Fragen D 4. bis D 6. gleichen denen von $B B$ und $B G$. Da in FOX keine dritte Entität eingeführt wird, wie in den anderen Geschichten, ist es der Vogel selbst, der hinter dem Fuchs herfliegt und ihn in den Schwanz beißt. Frage D 7. lautet in FOX, Wharum beißt der Vogel dem Fuchs in den Schwanz/Rücken?“ bzw. „Warum fliegt der Vogel dem Fuchs hinterher?“, ähnlich, wie in $B B$,Warum packt der Hund die Katze am Schwanz?“ und in $B G$,Warum beißt der Vogel den Fuchs in den Schwanz?" gefragt wird. Frage D 10. kann aus semantischen Gründen nicht danach gestellt werden, wen die Fischgräte nun lieber hat. Die ToM-Frage, ob das Kind die Geschichte als Ganzes verstanden habe, wurde daher stark modifiziert. Es wird gefragt, wie die Geschichte weiter gehen könnte und ob der Fuchs den Fisch vielleicht doch noch einmal bekomme. Im Verständnisteil kann das Kind, ebenso wie bei $B B$ und $B G$, zehn Punkte erreichen. FOX ermöglicht also eine fast deckungsgleiche Befragung der $S S$, der $S C$, der $I S T$ sowie der Comprehension.

\subsection{Testpersonen}

Die hier vorgestellte Vergleichsstudie ist konzipiert für Kinder im Alter zwischen fünf Jahren und dem Schuleintritt mit spätestens sieben Jahren. In Anlehnung an Sprachstandserhebungen wie beispielsweise HAVAS 5 (Reich \& Roth, 2004) steht diese Altersspanne im Fokus, da in Vorbereitung auf die Schulzeit eine Einschätzung des Sprachstandes erfolgt. Vor Beginn der ersten Tests wurde mit den Erziehern über den Sprachgebrauch und das Sozialverhalten der Kinder gesprochen und sich versichert, dass keines der teilnehmenden Kinder 
verhaltensauffällig oder sprachentwicklungsverzögert ist. Der Sprachstand der Kinder entspreche der Norm (TD $\left.{ }^{1}\right)$ und sie seien weder hörgeschädigt, sodass sie u.U. die Fragen der Kontrollperson akustisch nicht verstünden, noch sei bei einem von ihnen $A D H S$ oder Trisomie 21 diagnostiziert.

Bei den teilnehmenden Kindern handelt es sich sowohl um monolingual deutschsprachige, bilinguale und tri- bzw. multilinguale Kinder im Alter zwischen 5;01 bis 6;09. Insgesamt haben 33 Kinder an der Studie teilgenommen, von 27 Kindern (vierzehn Jungen und dreizehn Mädchen) konnten die Daten ausgewertet werden.

\section{Testergebnisse}

Die erzählten Geschichten der Kinder werden anhand der für die MAIN-Studie erstellten Protokollbögen und dem neu erstellten FOX-Protokollbogen evaluiert. Schließt man den Einfluss der Farbgestaltung der Bilder aus und vereinheitlicht die Durchführung der Tests, verbleiben Parameter, die für die Produktion von Narrativen bedeutsam sind und die explizit untersucht werden. Besonderes Augenmerk liegt auf der strukturellen Komplexität der Geschichten, die anhand der Protokollbögen mit den Bereichen A $S S$ und B $S C$ erfasst werden. Zunächst werden die Aufzeichnungen von $B B$ und $B G$, die mit einem kontrollierten Bildkonzept ausgestattet sind, mit denen von $C A T$ und $F O X$ verglichen, die nicht über diese makrostrukturelle Bearbeitung verfügen.

\subsection{Cat \& Fox Story vs. Baby Birds \& Baby Goats Geschichte}

Wie bereits beschrieben, werden anhand des Bewertungsbogens im Abschnitt I: Produktion A SS Punkte für die richtige Antwort bzw. erfolgte Erwähnung des Settings, der Goals, Attempts und Outcomes sowie der IST durch das Kind vergeben. Das Gesamtergebnis (Tabelle 1) zeigt, dass diese einen höheren Wert bei den Geschichten mit kontrolliertem Bildaufbau, also $B B$ und $B G$, aufweist (Wert: $170 \bumpeq 37 \%$ der maximal erreichbaren 459 Punkte) als bei den Geschichten ohne diese Struktur, also CAT und FOX (Wert: $145 \hat{=} 33 \%$ der maximal erreichbaren 433 Punkte). Die geringere maximal zu erreichende Punkteanzahl von 15 bei FOX im Vergleich zu 17 Punkten bei den anderen Geschichten wurde in der Berechnung berücksichtigt. Das Ergebnis ist unabhängig von Testtermin, Alter und Geschlecht der Kinder. Diese Daten lassen vermuten, dass die Geschichten mit kontrolliertem Bildaufbau auch einen höheren Wert im Bereich der $S C$ zur Folge haben. Die Ergebnisse in diesem Bereich weisen jedoch keine signifikanten Unterschiede auf: $\mathrm{p}$-Wert $=0,091, \mathrm{~F}(3,51)=2,28$. Insgesamt haben

$1 \mathrm{TD}=$ Typically-developing. 
sieben Kinder Geschichten mit einer kompletten $G A O$-Struktur bei $C A T$ und $F O X$ erzählt und nur sechs Kinder haben dies bei $B B$ und $B G$ getan. Ein Kind hat bei beiden Geschichten mindestens eine vollständige $G A O$-Struktur produziert. Folglich kann zunächst nicht davon ausgegangen werden, dass eine kontrollierte Makrostruktur signifikante Unterschiede in der Produktion kompletter GAOStrukturen verursacht. Aus den Ergebnissen kann man außerdem erkennen, dass die getesteten Kinder bei $B B$ und $B G$ mehr einzelne Attempts (59 gegenüber 46) und Outcomes (51 gegenüber 41) benannt haben, bei CAT und FOX aber mehr Goals (26 gegenüber 19) und IST (83 gegenüber 80).

Tabelle 1: Gesamtergebnis der Baby Birds \& Baby Goats Geschichten hinsichtlich Story Structure, Strucural Complexity, Goal, Attempt, Outcome und Internal State Terms im Vergleich zu Cat \& Fox Story

\begin{tabular}{lcc}
\hline & $\begin{array}{c}\text { Baby Birds \& Baby } \\
\text { Goats GESAMT }\end{array}$ & $\begin{array}{c}\text { CAT \& FOX Story } \\
\text { GESAMT }\end{array}$ \\
\hline Story Structure & 170 & 145 \\
Structural Complexity & 6 & 7 \\
Goal & 19 & 26 \\
Attempt & 59 & 46 \\
Outcome & 51 & 41 \\
Internal State Terms & 80 & 83 \\
\hline
\end{tabular}

\subsection{Der Testtermin}

Der Vergleich beider Testtermine erfolgt aus der Annahme heraus, dass Kinder beim zweiten Testtermin ein besseres Ergebnis erzielen, da sie dann bereits sowohl mit dem Testverfahren, der Art der Bildergeschichten als auch mit der Kontrollperson vertraut sind. Stellt man die Ergebnisse der beiden Termine einander gegenüber (siehe Tabelle 2), zeigt sich, dass die erzählten Geschichten ähnlich komplexe Strukturen aufweisen. Anhand der ausbalancierten Testliste konnten die Kinder beim ersten Testtermin insgesamt 445 Punkte erreichen, beim zweiten Termin 447. Der erreichte Wert der SS liegt beim ersten Termin bei 147 Punkten, beim zweiten Termin bei 168 Punkten. Damit liegt der Wert des ersten Testtermins bei 33\% der maximal erreichbaren SS Punktzahl, beim zweiten bei $37 \%$. Bei der Berechnung wurde die Verteilung der jeweiligen FOX-Geschichten des ersten und zweiten Testtermins und der damit verbundenen geringeren Maximalpunktzahl einkalkuliert.

Beim ersten Termin haben sechs Kinder eine komplette GAO-Struktur produziert, beim zweiten waren es sieben Kinder. Obwohl es plausibel erscheint, dass sich die Ergebnisse der beiden Testtermine unterscheiden sollten, zeigt sich 
jedoch anhand des Testverfahrens der Linearen Regression kein signifikanter Unterschied: $\mathrm{p}$-Wert $=0,103, \mathrm{df}=48, \mathrm{t}-$ Wert $=1,659$.

Tabelle 2: Vergleich der Testtermine hinsichtlich Story Structure und GAO-Strukturen

\begin{tabular}{lcc}
\hline & 1. Testtermin & 2. Testtermin \\
\hline Story Structure & 147 & 168 \\
GAO-Strukturen & 6 & 7 \\
\hline
\end{tabular}

\subsection{Der Einfluss des Alters}

Mit zunehmendem Alter steigt bei $T D$-Kindern auch die Sprachkompetenz. Daher werden nun die Daten hinsichtlich der chronologischen Altersreihenfolge der Kinder betrachtet. Die Frage ist, ob Kinder mit zunehmendem Alter stärker auf eine makrostrukturell kontrollierte Bildoberfläche reagieren und sich dies in der $S S$ oder der $S C$ ihrer erzählten Geschichten niederschlägt. Graphik 1 visualisiert die steigenden Werte der SS mit zunehmendem Alter der Kinder.

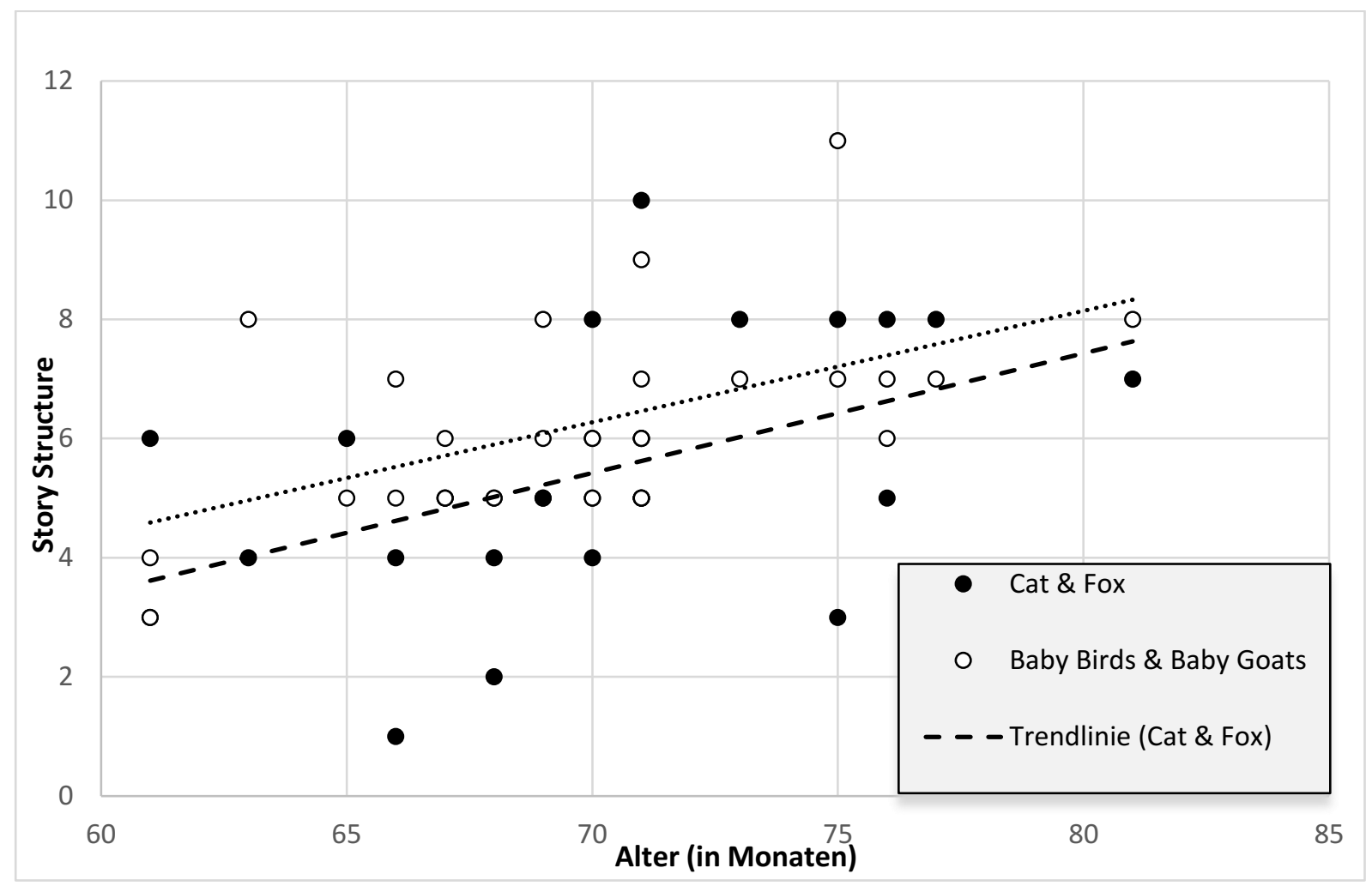

Graphik 1: Entwicklung von Story Structure bei Baby Birds und Baby Goats Geschichten im Vergleich zu Cat und Fox Story mit zunehmendem Alter

Fraglich ist jedoch, ob die Ergebnisse der Geschichten mit GAO-Struktur signifikant über denen der Geschichten ohne GAO-Struktur liegen. Wie die Lineare Regressionsberechnung zeigt, ist der Unterschied der beiden Geschichten signifikant, wenn man annimmt, dass der Alterseffekt in beiden Geschichten 
gleich ist: $\mathrm{p}$-Wert $=0,041, \mathrm{t}$-Wert $=-2,093$. Die Produktion vollständiger $G A O$ Strukturen beginnt bei den Probanden (mit einer Ausnahme) ab fünf Jahren und neun Monaten (69 Monaten). Dies trifft sowohl auf $C A T$ und $F O X$ als auch auf $B B$ und $B G$ gleichermaßen zu.

\subsection{Die einzelnen Geschichten}

Neben der Tatsache, dass $B B$ und $B G$ im Vergleich zu $C A T$ und $F O X$ mit einem kontrollierten Bildkonzept ausgestattet sind, variieren sie zusätzlich in ihrem Handlungsverlauf und den Protagonisten. Unter Umständen können auch diese Parameter die Komplexität der von den Kindern erzählten Geschichten beeinflussen. Deshalb wurden die Geschichten einzeln auf die produzierten GAOStrukturen hin untersucht. Das Ergebnis dieser Auswertung zeigt Graphik 2.

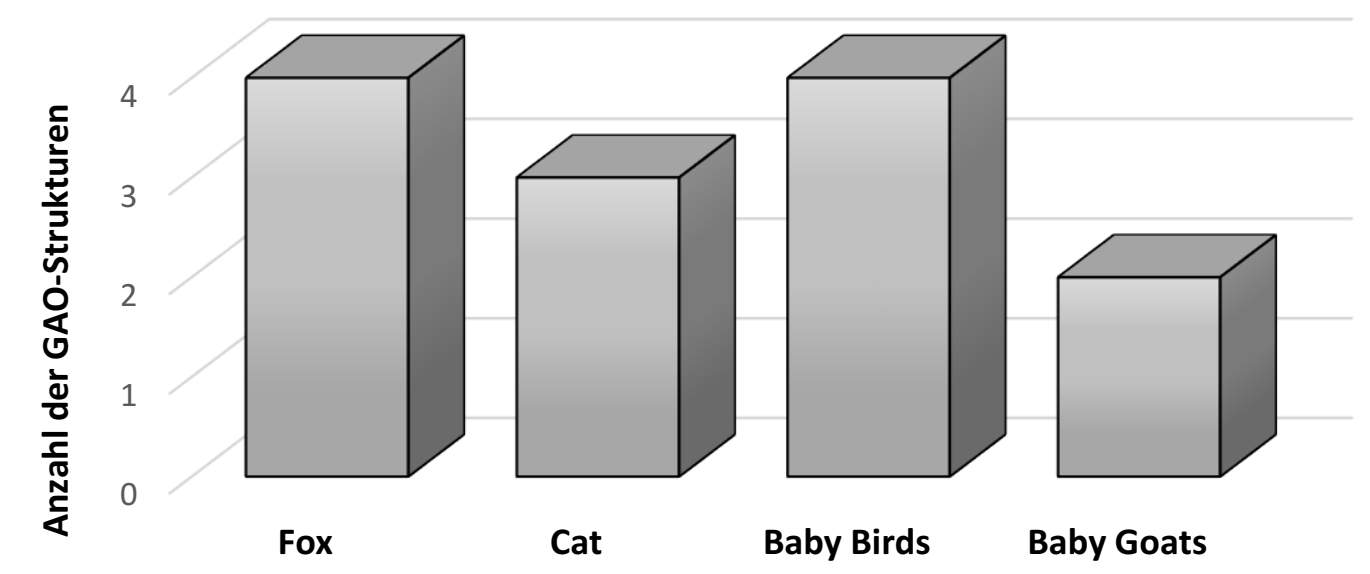

Graphik 2: Verteilung der produzierten GAO-Strukturen

$F O X$ wurde von zwei Jungen und zwei Mädchen mit kompletter $G A O$-Struktur erzählt. Hierbei lag die Altersspanne zwischen 70 und 77 Monaten. Beim Testtermin war keine eindeutige Präferenz zu erkennen, da ein Kind (weiblich, 73 Monate) $F O X$ zum ersten, die anderen Kinder zum zweiten Termin erzählten. Dies lässt dementsprechend vermuten, dass die $G A O$-Struktur unabhängig von Testtermin, Alter und Geschlecht der Kinder produziert wird. $C A T$ haben drei Jungen im Alter von 69, 71 und 81 Monaten mit vollständiger $G A O$-Struktur produziert. Zweimal geschah dies zum ersten und einmal zum zweiten Termin. $B B$ wurde, wie $F O X$, von vier Kindern, zwei Jungen und zwei Mädchen, mit kompletter $G A O$-Struktur erzählt. Die Altersspanne reicht von 63 Monaten bis hin zu 77 Monaten. Die beiden Jungen schilderten $B B$ zum ersten Termin, die beiden Mädchen zum zweiten. $B G$ wurde von zwei Mädchen im Alter von 71 und 76 Monaten mit kompletter $G A O$-Struktur geschildert. Einmal wurde diese zum ersten und einmal zum zweiten Termin erzählt. 
Aufgrund der dargestellten Ergebnisse scheinen weder die Makrostruktur der Bildergeschichten selbst, noch Faktoren wie Testtermin, Alter und Geschlecht der Kinder dafür relevant zu sein, ob die Kinder eine Geschichte mit kompletter $G A O$-Struktur erzählen, oder nicht.

\subsection{Das Geschlecht der Probanden}

Neben den demographischen Variablen der sozialen Schicht und des elterlichen Bildungsstandes hat auch das Geschlecht einen Einfluss auf die Sprachentwicklung des Kindes. Korreliert jedoch eine schnellere Sprachentwicklung bei Mädchen, hinsichtlich Wortschatz und Grammatik, gegenüber Jungen mit der Produktion komplexerer Geschichten? (Szagun, 2006:228). Graphik 3 zeigt eine Gegenüberstellung der Bildergeschichten mit und ohne kontrolliertem Bildkonzept hinsichtlich der Geschlechter der Probanden. Hierbei kann man erkennen, dass fünf Jungen eine komplette $G A O$-Struktur bei den Geschichten ohne kontrolliertem Bildkonzept (CAT und $F O X)$ erzählten, wohingegen dies nur zwei Jungen bei den Geschichten mit diesem Konzept ( $B B$ und $B G$ ) getan haben. Ein Junge erzählte in beiden Geschichten eine komplette GAO-Struktur.

Bei Mädchen sieht man eine entgegengesetzte Tendenz. Zwei von ihnen haben eine Geschichte erzählt, die eine $G A O$-Struktur aufweist, ohne dass die Bildergeschichte mit kontrolliertem Bildkonzept ausgestattet ist (CAT und $F O X$ ). Vier Mädchen haben dies bei den Geschichten mit diesem Konzept ( $B B$ und $B G$ ) getan.

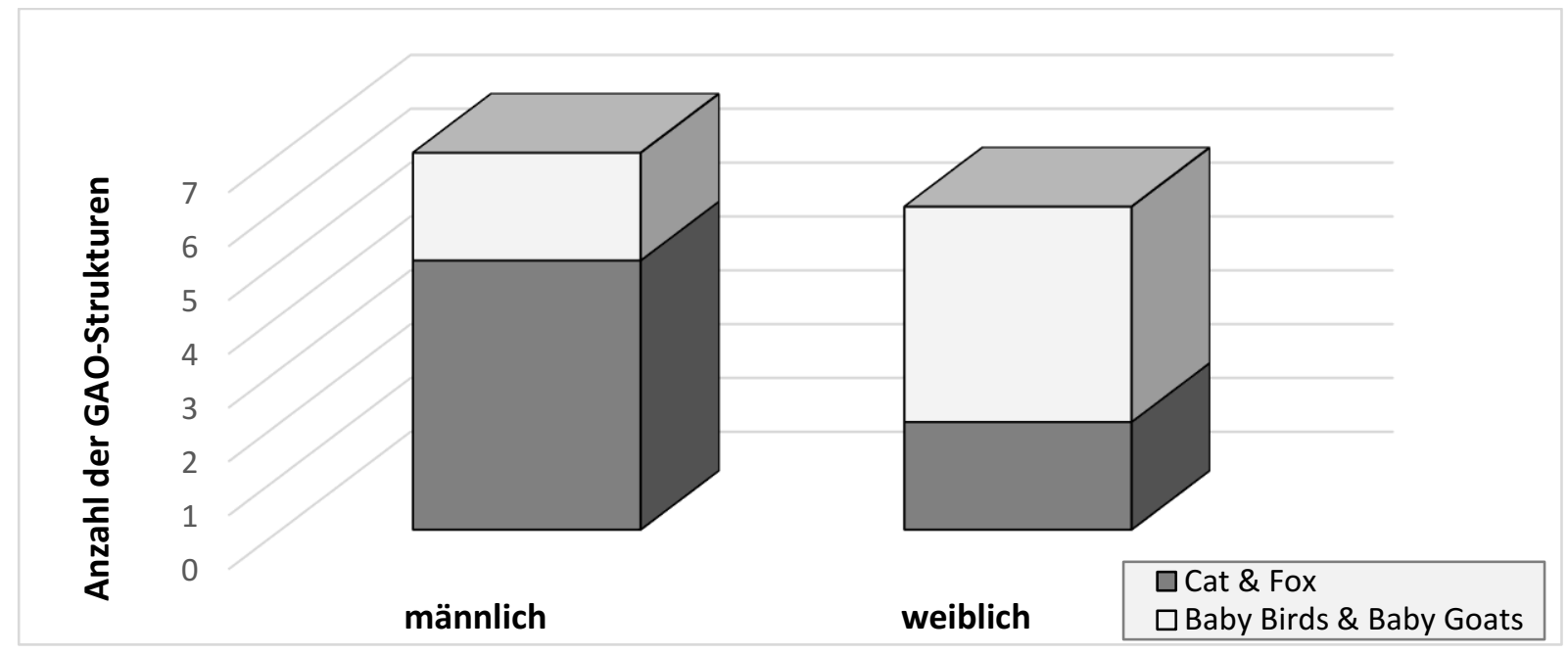

Graphik 3: Verteilung produzierter $G A O$-Strukturen nach Geschlechtern

Vergleicht man die Angaben der Graphik, lässt sich feststellen, dass Jungen häufiger bei Geschichten ohne kontrolliertem Bildaufbau eine höhere Komplexitätsstufe $(S C)$ erreichten. Bei Mädchen ist dies bei den 
Bildergeschichten mit diesem Konzept der Fall. Der Fisher-Test zeigt jedoch kein signifikantes Ergebnis: p-Wert =0,286.

\subsection{Resümee der Datenauswertung}

Die Datenauswertung deutet an, unter welchen Bedingungen ein makrostrukturell kontrollierter Bildaufbau Einfluss auf die Erzählfähigkeiten von Vorschulkindern ausüben könnte. Bei dem Vergleich von $C A T$ und $F O X$ als Geschichten ohne $G A O$-Struktur gegenüber $B B$ und $B G$ mit GAO-Struktur war zwar ein höherer $S S$ Wert zu erkennen, der jedoch keinen Einfluss auf die produzierten $G A O$ Strukturen hatte.

Bei der Untersuchung der Ergebnisse beider Testtermine ist ein Unterschied des SS Wertes zwar plausibel, der nach statistischer Berechnung jedoch nicht signifikant ist. Betrachtet man die Daten vor dem Hintergrund der chronologischen Altersreihenfolge der getesteten Kinder, lässt sich zunächst feststellen, dass die Komplexität des Geschichtsaufbaus mit ansteigendem Alter zunimmt. Diese Tendenz trifft auf beide Arten von Geschichten zu, unabhängig davon, ob ihre Bilder makrostrukturell kontrolliert sind oder nicht. Aber darüber hinaus ist nach Berechnung der Linearen Regression der Unterschied zwischen den Geschichten zudem auch signifikant.

Bei der Betrachtung der einzelnen Bildergeschichten zeichnen sich keine eindeutigen Präferenzen hinsichtlich einer bestimmten Geschichte oder eines makrostrukturellen Konzepts ab. $B B$ und $F O X$ weisen die meisten produzierten $G A O$-Strukturen auf, obwohl nicht beide mit einem kontrollierten Bildaufbau versehen sind. Dennoch haben die Kinder dieser Studie vergleichbare Geschichten hinsichtlich ihrer SC erzählt. Grundsätzlich muss allerdings vermerkt werden, dass ein Fehlen von signifikanten Unterschieden in den Daten kein Beweis dafür ist, dass eine $G A O$-Struktur keinen Einfluss auf die Produktion und Perzeption von Bildergeschichten ausübt.

Die Auswertung der Daten lässt einen Unterschied in der Produktion von $G A O$-Strukturen hinsichtlich der Geschlechter vermuten. Danach haben Jungen häufiger $C A T$ und $F O X$ mit einer vollständigen $G A O$-Struktur erzählt, als $B B . B G$ hat kein Junge mit vollständiger $G A O$-Struktur geschildert. Bei Mädchen ist eine entgegengesetzte Situation erkennbar. Vier von ihnen haben $B B$ und $B G$ mit kompletter $G A O$-Struktur erzählt, wohingegen nur zwei Mädchen dies bei $F O X$ getan haben. $C A T$ hat kein Mädchen mit kompletter GAO-Struktur erzählt. Auch wenn der Fisher-Test keine Signifikanz bezüglich des Geschlechts der Kinder ergab, eröffnen die Ergebnisse dieser Studie die Frage danach, ob es Unterschiede in der Produktion von GAO-Strukturen zwischen Jungen und Mädchen gibt. 


\section{Schlussbetrachtungen}

Da anhand beider Formen von Narrativen Kinder auf ihre Sprachentwicklung und Erzählkompetenzen hin untersucht werden, war es das Anliegen dieser empirischen Studie, Kinder jeweils eine Geschichte mit kontrolliertem Bildaufbau (Baby Birds oder Baby Goats Geschichte) und eine ohne dieses Konzept (Cat Story oder Fox Story) erzählen zu lassen und die Ergebnisse miteinander zu vergleichen. Hinsichtlich der Story Structure und Structural Complexity unterscheiden sich die Ergebnisse statistischer Berechnungen zufolge nicht signifikant. Ebenso wies die Gegenüberstellung der Ergebnisse beider Testtermine keine signifikanten Unterschiede auf. Betrachtet man jedoch die Entwicklung der Erzählkomplexität mit zunehmendem Alter der Kinder, ist zum einen ein Anstieg der Structural Complexity zu erkennen. Darüber hinaus ist zusätzlich auch der Unterschied zwischen beiden Formen von Geschichten mit und ohne GAO-Struktur signifikant. Bildergeschichten, die mit einem makrostrukturell kontrollierten Bildaufbau ausgestattet sind, lassen Kinder mit zunehmendem Alter demnach komplexere Geschichten erzählen, als Bildergeschichten ohne diese Struktur.

Nach Abschluss der Untersuchungen dieser Studie lässt sich erkennen, dass das zunehmende Alter der Kinder einen signifikanten Einfluss auf die Produktion von komplexen Geschichten hat. Geschichten, welche Kinder aufgrund einer Bildergeschichte, die mit einem makrostrukturell kontrollierten Bildaufbau versehen ist, erzählen, weisen darüber hinaus auch eine signifikant höhere Komplexität auf, da mehr Goals, Attempts und Outcomes verbalisiert werden.

\section{$7 \quad$ Literaturverzeichnis}

Gagarina, N., Klop, D., Kunnari, S., Tantele, K., Välimaa, T., Balčiūnienè, I., Bohnacker, U., \& Walters, J. (2012). MAIN: Multilingual Assessment Instrument for Narratives. ZAS Papers in Linguistics 56, 1-140.

Gülzow, I. \& Gagarina, N. (2007). Intersentential Pronominal Reference in Child and Adult Language. Proceedings of the Conference on Intersentential Pronominal Reference in Child and Adult Language. ZAS Papers in Linguistics 48, 203-223.

Hickmann, M. (2002). Children's Discourse. Person, Space and Time across Languages. Cambridge: Cambridge University Press.

Kuehnast, M. (2006). Developing a Corpus for Contrastive Studies of Intersentential Anaphora in Child Language. In: A. Gerd (ed.) Proceeding of the International Conference "Corpus Linguistics 2006", October $10-14^{\text {th }}, 2006$. St. Petersburg: University Press, 224-225. 
Reich, H.H., Roth, H.-J. (2004). Das Hamburger Verfahren zur Analyse des Sprachstands bei Fünfjährigen - HAVAS 5. Hamburg: Landesinstitut für Lehrerbildung und Schulentwicklung Hamburg.

Schneider, P., Dubé, R. V., \& Hayward, D. (2005). The Edmonton Narrative Norms Instrument. http://www.rehabresearch.ualberta.ca/enni

Schneider, P., Rivard, R. \& Debreuil, B. (2011). Does Colour Affect the Quality or Quantity of Children's Stories Elicited by Pictures. Child Language Teaching and Therapy 27(3), 371-378.

Stein, N. \& Glenn, C. (1979). An Analysis of Story Comprehension in Elementary School Children. In: R. Freedle (ed.) New Directions in Discourse Processing, 58-120.

Szagun, G. (2006). Sprachentwicklung beim Kind. Weinheim/Basel: Beltz Verlag.

Thorndyke, P.W. (1977). Cognitive Structures in Comprehension and Memory of Narrative Discourse. Cognitive Psychology 9, 77-110.

Tomasello, M. (2003). Constructing a Language. A Usage-Based Theory of Language Acquisition. Cambridge MA: Harvard University Press.

Trabasso, T. \& Nickels, M. (1992). The Development of Goal Plans of Action in the Narration of a Picture Story. Discourse Processes 15, 249-275.

Westby, C.E. (2005). Assessing and Remediating Text Comprehension Problems. In: H. Catts, A. Kamhi (eds.) Language and Reading Disabilities. Boston, MA: Allyn \& Bacon. 